\title{
A flourishing field: going back to the roots of the Archaea
}

\author{
This issue marks the 40th anniversary of the publication of the landmark article by Carl Woese \\ and George Fox in which they proposed the Archaea as a third domain of life in addition to the \\ Eukarya and Bacteria.
}

The tree of life is a metaphor first used by Charles Darwin that expresses that all life on Earth is related by common descent. In 1977, Woese and Fox proposed that the tree of life is divided into three branches, or 'urkingdoms' - the urkaryotes, eubacteria and archaebacteria, which correspond to the current terms eukaryotes, bacteria and archaea. Although a three-domain tree was controversial to some, this study was soon accepted and is widely regarded as one of the most important discoveries in biology of the past century. Woese and Fox's work inspires scientists to this day, as showcased in an essay by leading archaeal scientists on the legacy of Woese ${ }^{1}$. At the time of their discovery, very little was known about what makes archaea distinct from bacteria. However, recent progress has led to a sea change in our understanding of the fundamental biology, evolution, metabolic versatility and ecological impact of archaea. Recognizing the importance of these discoveries, Nature Reviews Microbiology has published several articles (see Collection on Archaea and the tree of life and some of the highlights below) that explore the advances that have been made over the past 40 years in the field.

In the original landmark paper ${ }^{2}$, Woese analysed $16 \mathrm{~S}$ ribosomal RNA (rRNA) genes from just four methanogenic archaea to conclude that cellular life is divided into three evolutionary lineages. These genes remain at the heart of bacterial and archaeal taxonomy; for an example of how powerful this technique is, see an Analysis article by Rosselló-Móra and colleagues that we published in 2014 (REF. 3). Today, thousands of archaeal species have been identified in diverse environments, ranging from deep-sea hydrothermal vents to the human gut. Innovations in DNA sequencing technologies and culture-independent metagenomics have been instrumental in informing our view of the genes that are encoded in archaeal genomes and the evolution of archaea. In an Opinion article, López-García et al. ${ }^{4}$ propose the intriguing possibility that mesophilic archaea adapted to their environments through the horizontal acquisition of genes from bacteria. The exchange of genes is a major driving force for evolution across the tree of life and has a role in archaeal speciation, adaptation and maintenance of diversity. The importance of horizontal gene transfer (HGT) in archaeal evolution was recently reviewed by Albers and colleagues ${ }^{5}$.

The study of the Archaea is helping us make headway in understanding microbial ancestry and the origins of all multicellular life on Earth. The rise in cultureindependent metagenomics and the expansion of available archaeal genomes has resulted in the proposal of several new phyla and three superphyla - the DPANN, TACK and Asgard superphyla. Importantly, the identification of members of these superphyla has revealed unprecedented insights into the evolution of eukaryotes and has led to the revival of a two-domain theory of the tree of life (see Collection). On page $\underline{711}$, Ettema and colleagues revisit central questions concerning eukaryogenesis in light of the discovery of new members of the Archaea. Whether a two-domain or three-domain tree holds true, the recent discovery of the Asgard archaea that possess genes that were once thought to be unique to eukaryotes, such as components of the vesicular trafficking machinery, provides exciting evidence for an archaeal ancestor of eukaryotes. One of the most remarkable features of archaea is the diversity of their viruses. On page 724, Prangishvili and colleagues discuss the extraordinary biology of archaeal viruses.

Despite 40 years of research and many groundbreaking discoveries, numerous questions remain to be answered. What is the precise relationship between archaea and eukaryotes? What is the basis for the physiological diversity of archaea? What is the ecological role of archaea? We hope that our Collection will guide and inspire future research, new perspectives and possible answers to long-standing questions.

\footnotetext{
1. Albers, S.-V., Forterre, P., Prangishvili, D. \& Schleper, C. The legacy of Carl Woese and Wolfram Zillig: from phylogeny to landmark discoveries. Nat. Rev. Microbiol. 11, 713-719 (2013).

2. Woese, C. R. \& Fox, G. E. Phylogenetic structure of the prokaryotic domain: the primary kingdoms. Proc. Natl Acad. Sci. USA 74 5088-5090 (1977).

3. Yarza, P. et al. Uniting the classification of cultured and uncultured bacteria and archaea using 16S rRNA gene sequences. Nat. Rev. Microbiol. 12, 635-645 (2014).

4. López-García, P., Zivanovic, Y., Deschamps, P. \& Moreira, D. Bacterial gene import and mesophilic adaptation in archaea. Nat. Rev. Microbiol. 13, 447-456 (2015).

5. Wagner, A. et al. Mechanisms of gene flow in archaea. Nat. Rev. Microbiol. 15, 492-501 (2017).
} 